\title{
Simulação do Processo de Cristalização usando Métodos Numéricos Estocásticos
}

\section{Simulation of Crystallization Process using Stochastic Numerical Methods}

Fran Sérgio Lobatoํㅜ Fabiano Fortunato Teixeira dos Santos²

\section{RESUMO}

Tradicionalmente, durante a simulação de sistemas de Engenharia e áreas afins, não se considera a presença de incertezas nas variáveis, nos parâmetros ou nos modelos que caracterizam o fenômeno em análise. Nesse contexto, deixa-se de avaliar a influência dessas incertezas nos perfis obtidos. Desde o ano de 1965, o interesse pela resolução de modelos estocásticos tem atraído a atenção da comunidade científica, visto que todo modelo fenomenológico apresenta, no mínimo, incerteza associada às hipóteses consideradas durante sua formulação. Diante do que foi apresentado, este trabalho tem como objetivo simular um processo de cristalização em que são consideradas incertezas na concentração de cristais. Para essa finalidade, consideram-se vários métodos numéricos estocásticos para a resolução do modelo matemático (balanço de massa e balanço populacional) que representa este processo. Inicialmente, estes métodos são aplicados a dois problemas matemáticos clássicos que apresentam solução analítica. A partir dos resultados obtidos, observase a qualidade da solução em função da qualidade do método numérico estocástico considerado.

Palavras-chave: Processo de Cristalização, Modelo Estocástico, Métodos Numéricos.

\section{ABSTRACT}

Traditionally, during the simulation of engineering systems and related areas, no kind of uncertainties in variables, parameters or models that characterize the analyzed phenomenon are considered. In this context, the influence of these uncertainties on obtained profiles is not investigated. Since the year 1965, the interest in solving stochastic models has attracted the attention of scientific community. This is due to presence of uncertainties associated with the hypotheses considered during the formulation of phenomenological models. In this context, this contribution aims to simulate a crystallization process in which the model presents uncertainty in crystals concentration. For this purpose, some stochastic numerical methods are considered to solve the mathematical model (mass and population balances) that represents this process. Initially, these methods are applied in two classical mathematical functions that present analytical solution. The results show that the quality of solution is a function of the stochastic numerical method considered.

Keywords: Crystallization Process, Stochastic Process, Numerical Methods.

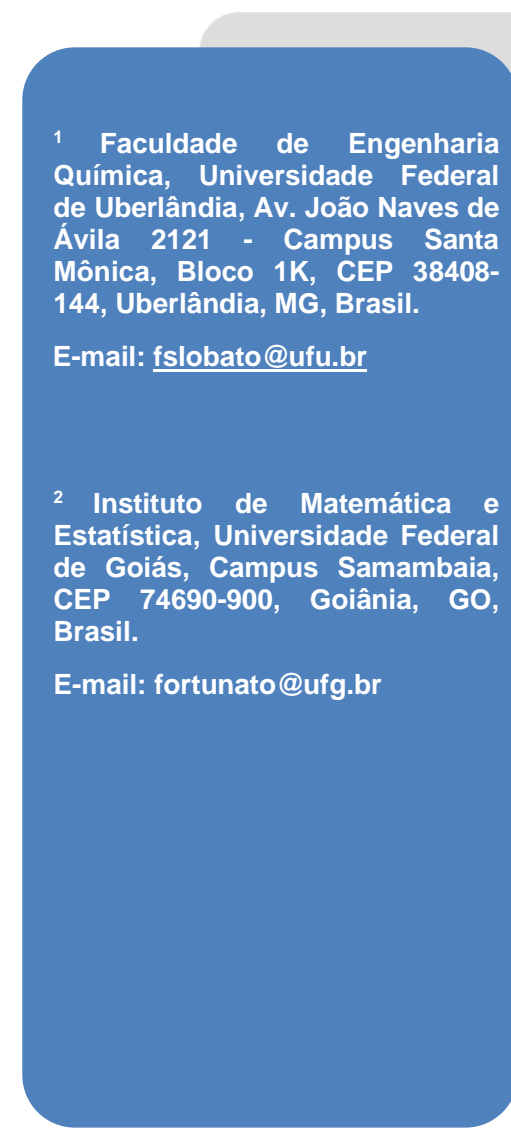




\section{INTRODUÇAOO}

O estudo de incertezas em modelos fenomenológicos tem se configurado como uma linha de pesquisa de grande interesse, o que se deve à necessidade de avaliar a presença de aleatoriedade associada às variáveis, aos parâmetros e aos próprios modelos desenvolvidos. Para isso, vários métodos podem ser utilizados, entre os quais os baseados em análise de robustez e confiabilidade. Para essas duas classes de metodologias, a priori, nenhuma informação sobre incerteza associada aos modelos é considerada. Neste caso, para avaliar a influência de perturbações em modelos, pode-se utilizar as Equações Diferenciais Estocásticas (EDEs).

$\mathrm{Na}$ literatura especializada, inúmeras aplicações considerando EDEs podem ser encontradas, como estudos de caso das áreas de Economia, Biologia e Medicina (Carletti, 2006; Ivanov e Swishchuk, 2008; Lo, 2010). Em linhas gerais, pode-se dizer que os modelos estocásticos oferecem uma representação mais realista de sistemas físicos, químicos e biológicos, em comparação com modelos determinísticos (nenhuma informação sobre incertezas é considerada), visto os erros que surgem durante a modelagem e os experimentos relacionados ao fenômeno em análise.

Em Engenharia Química, um dos processos em que se pode observar a presença de incertezas é o de cristalização; uma operação unitária que tem como finalidade a obtenção de material particulado de alta pureza, com grande aplicabilidade em processos químicos e farmacêuticos. Essa técnica é considerada como um método para a separação de misturas de substâncias representadas por materiais crus ou por produtos de reação.

A cristalização pode ocorrer com formação de partículas, como a solidificação de um líquido, ou com formação de sólidos dispersados em uma solução, classificado em três fases. A primeira fase é a geração da força motriz, ocasionada pela supersaturação, que ocorre quando se consegue quantidade de soluto superior à quantidade de saturação, sem que ocorra a precipitação do soluto. A segunda fase é a nucleação, que influencia significativamente o tamanho dos cristais, a qual pode não existir quando se opera na região metaestável. Na última fase da cristalização, o principal fenômeno envolvido é o crescimento dos cristais, que envolve o transporte de massa do soluto de uma solução para a superfície do cristal (Saito et al. 2002).

Matematicamente, o fenômeno de cristalização pode ser formulado por um sistema integro-diferencial, que modela o balanço da população de cristais, o balanço de massa e 
o balanço de energia, associado a equações constitutivas que representam as taxas de crescimento e nucleação de cristais (Myerson, 1952). Do ponto de vista prático, essas equações constitutivas apresentam parâmetros que devem ser estimados para um conjunto de condições avaliadas experimentalmente.

Diante do que foi apresentado, esse trabalho tem por objetivo avaliar a influência de incertezas no processo de produção de cristais de sulfato de potássio. Para tanto, considera-se a presença de incerteza na concentração de cristais. O modelo integrodiferencial é transformado em um equivalente puramente diferencial via aplicação do Método dos Momentos. Esse sistema diferencial é integrado, considerando diferentes abordagens numéricas estocásticas.

Este trabalho está estruturado da seguinte forma: a seção 2 apresenta o modelo matemático que caracteriza o processo de cristalização; a seção 3 apresenta uma revisão sobre alguns métodos numéricos estocásticos empregados para resolver EDEs de primeira ordem. A metodologia proposta neste trabalho é apresentada na seção 4; os resultados obtidos com a aplicação da metodologia proposta em problemas matemáticos e no processo de cristalização são apresentados na seção 5; e, finalmente, as conclusões são apresentadas na última seção.

\section{MODELAGEM MATEMÁTICA DO PROCESSO DE CRISTALIZAÇÃO}

A modelagem matemática do processo de cristalização consiste na associação entre os balanços de massa e populacional (distribuição de tamanho de cristais), já que nessa operação unitária é produzida uma massa discreta de partículas de vários tamanhos. A seguir, são apresentadas as equações que descrevem o processo de cristalização em análise.

\subsection{Balanço de Massa}

O balanço material do soluto é realizado com base no fato de que uma mudança de concentração resulta em uma variação da massa de cristais por unidade de volume. Neste caso, o balanço de massa para o soluto que está sendo cristalizado e para o solvente no cristalizador pode ser representado pelo seguinte modelo matemático (Myerson, 1952; Costa, 2003): 


$$
\frac{d C}{d t}=\frac{M_{f o}}{M}\left(C_{f o}-C\right)-3 \rho_{C} k_{v} \int_{0}^{\infty} G(L, t) n(L, t) L^{2} d L
$$

em que $C$ é a concentração de cristais; $t$ é o tempo de operação; $L$ é a dimensão característica do cristal; $M$ é a massa do solvente no cristalizador; $M_{f o}$ e $C_{f o}$ representam o fluxo mássico de solvente e a concentração de soluto, respectivamente; $\rho_{c}$ é a densidade do cristal; $k_{v}$ é o fator de forma referente ao volume; $G$ é a taxa de crescimento dos cristais; e $n$ é a função distribuição de tamanho de cristais.

\subsection{Balanço Populacional}

Matematicamente, a população de cristais pode ser representada por uma função que descreve a distribuição (em número, massa ou volume) dos mesmos. Uma população que tenha um grande número de cristais pequenos e um pequeno número de cristais bastante grandes pode ter sua representação em número com um aspecto muito diferente da representação dessa mesma população em massa, pois grande parte da massa da população pode estar localizada na parte da população dos maiores cristais (Costa, 2003).

A distribuição do tamanho dos cristais pode ser prevista a partir do balanço do número de partículas. Para isso, as equações de balanço de população são empregadas para descrever a forma como a distribuição de tamanho de uma população de cristais se desenvolve no tempo como resultado de vários processos cinéticos (Rawlings et al. 1993; Rawlings et al, 2001). Genericamente, a equação geral para o balanço de densidade em número de um cristalizador tendo volume $V$ é dada por:

$$
\frac{\partial n}{\partial t}+\frac{\partial(G n)}{\partial L}+n \frac{\partial V}{V \partial t}+D(L)-B(L)+\sum_{k} \frac{V_{k} n_{k}}{V}=0
$$

onde o termo $\partial n / \partial t$ representa a mudança da densidade com relação ao tempo; $\partial(G n) / \partial L$ descreve a diferença entre cristais crescendo para dentro ou para fora do intervalo $d L$, devido à taxa de crescimento de cristal $G=d L / d t$; o termo $n(\partial V / V \partial t)$ considera mudanças no volume em relação ao tempo; os termos $D(L)$ e $B(L)$ representam as taxas de desaparecimento e aparecimento, respectivamente; e o último termo fornece a soma de todos os fluxos de partículas, entrando e saindo do cristalizador (Costa, 2003). 
É importante ressaltar que para uma completa descrição do modelo matemático do processo de cristalização, faz-se necessário utilizar informações sobre o perfil de temperatura, isto é; sobre o balanço de energia. Nesta contribuição, o modelo de temperatura será apresentado posteriormente.

Em resumo, o modelo descrito é formado por um sistema integro-diferencial que requer, para sua simulação, a utilização de equações constitutivas para os termos $B, D$ e G. Neste caso, faz-se necessário a determinação dessas equações via formulação e resolução de um problema inverso.

\section{METODOS NUMERICOS ESTOCÁSTICOS}

Na literatura especializada, vários métodos numéricos específicos para a integração de modelos estocásticos podem ser encontrados (Schaffter, 2009, Riadh, 2014). Nesta seção, são destacadas algumas metodologias para a integração de EDEs de primeira ordem representadas pelo processo de Itô (Oksendal, 1952):

$$
d X(t)=\mu(X(t)) d t+\sigma(X(t)) d W(t), \quad X(0)=X_{0}, \quad 0 \leq t \leq T
$$

em que o processo estocástico $\{W(t)\}_{t \geq 0}$, conhecido por processo de Wiener, está definido sobre um espaço de probabilidade adequado e é independente da variável aleatória $X_{0}$ para o tempo $t$ definido no intervalo [0 T]. Além disso, as funções $\mu$ e $\sigma$ são definidas no campo dos números reais. O termo $\mu(X(t)) d t$ é conhecido por drift, e é a parcela determinística da Equação (3). Já o termo $\sigma(X(t)) d W(t)$ é chamado de difusão e é responsável por introduzir estocasticidade na Equação (3); ou seja, introduzir incerteza na modelagem.

A seguir são apresentados os principais métodos numéricos propostos para resolver equações diferenciais estocásticas de primeira ordem.

\subsection{Euler-Maruyama}

O Método de Euler-Maruyama é uma aproximação numérica iterativa baseada no esquema proposto por Itô, representado como segue:

$$
X_{i+1}=X_{i}+\mu_{i} \Delta t+\sigma_{i} \Delta W_{i}
$$

em que $i$ é um contador; $\mu_{i}$ e $\sigma_{i}$ representam as avaliações dos termos determinístico e estocástico no ponto $X_{i}$, respectivamente; $\Delta t$ é o tamanho do passo de integração, 
definido como sendo $T / N$, onde $N$ é o número de pontos de discretização; e $\Delta W_{i}$ é a avaliação do processo estocástico no i-ésimo ponto.

\subsection{Euler-Heun}

O Método de Euler-Heun é uma versão melhorada do Método de Euler-Maruyama, em que o ponto $X_{i+i}$ é atualizado, considerado a avalição média entre os pontos $X_{i}$ e $Y_{i}$, conforme a seguinte relação:

$$
X_{i+1}=X_{i}+0,5\left(\mu_{i}\left(X_{i}\right)+\mu_{i}\left(Y_{i}\right)\right) \Delta t+\sigma_{i}\left(Y_{i}\right) \Delta W_{i}
$$

em que:

$$
Y_{i}=X_{i}+\mu_{i}\left(X_{i}\right) \Delta t+\sigma_{i}\left(X_{i}\right) \Delta W_{i}
$$

onde $\mu_{i}\left(X_{i}\right)$ e $\mu_{i}\left(Y_{i}\right)$ representam as avaliações dos termos determinísticos nos pontos $X_{i}$ e $Y_{i}$, respectivamente.

\subsection{Milstein}

O Método de Milstein é um esquema numérico que se diferencia da aproximação de Itô, sendo a sua fórmula iterativa, sem o uso de derivadas, representada por:

$$
X_{i+1}=X_{i}+\mu_{i}\left(X_{i}\right) \Delta t+\sigma_{i}\left(X_{i}\right) \Delta W_{i}+\frac{1}{2 \sqrt{\Delta t}}\left(\mu_{i}\left(Y_{i}\right)+\mu_{i}\left(X_{i}\right)\right)\left(\Delta W_{i}^{2}-\Delta t\right)
$$

em que:

$$
Y_{i}=X_{i}+\mu_{i}\left(X_{i}\right) \Delta t+\sigma_{i}\left(X_{i}\right) \sqrt{\Delta t}
$$

\subsection{Runge-Kutta 1,0 - Strong 1,0 Order Method}

Na tentativa de aumentar a qualidade da aproximação numérica considerada para a resolução da Equação (3), várias adaptações da tradicional família de Métodos RungeKutta foram propostas. A seguir, apresenta-se o Runge-Kutta 1,0.

$$
X_{i+1}=X_{i}+\mu_{i}\left(X_{i}\right) \Delta t+\sigma_{i}\left(X_{i}\right) \Delta W_{i}+0,5\left(\mu_{i}\left(Y_{i}\right)-\mu_{i}\left(X_{i}\right)\right) \frac{\left(\Delta W_{i}^{2}-\Delta t\right)}{\sqrt{\Delta t}}
$$

em que:

$$
Y_{i}=X_{i}+\sigma_{i}\left(X_{i}\right) \sqrt{\Delta t}
$$




\subsection{Runge-Kutta 1,5 - Weak 1,5 Order Method}

A relação que segue se diferencia da anterior pela presença do termo $\mu_{i}\left(X_{i}\right) \Delta t$ no cálculo do ponto $Y_{i}$.

$$
X_{i+1}=X_{i}+\mu_{i}\left(X_{i}\right) \Delta t+\sigma_{i}\left(X_{i}\right) \Delta W_{i}+0,5\left(\mu_{i}\left(Y_{i}\right)-\mu_{i}\left(X_{i}\right)\right) \frac{\left(\Delta W_{i}^{2}-\Delta t\right)}{\sqrt{\Delta t}}
$$

em que:

$$
Y_{i}=X_{i}+\mu_{i}\left(X_{i}\right) \Delta t+\sigma_{i}\left(X_{i}\right) \sqrt{\Delta t}
$$

\subsection{Runge-Kutta 2,0 - Weak 2,0 Order Method}

Finalmente, o Método de Runge-Kutta 2,0 é dado por:

$$
\begin{gathered}
X_{i+1}=X_{i}+\frac{1}{2}\left(\mu_{i}\left(U_{i}\right)+\mu_{i}\left(X_{i}\right)\right) \Delta t+\frac{1}{4}\left(\sigma_{i}\left(U_{i}^{+}\right)+\sigma_{i}\left(U_{i}^{-}\right)+2 \sigma_{i}\left(X_{i}\right)\right) \Delta W_{i}+ \\
+\frac{1}{4}\left(\sigma_{i}\left(U_{i}^{+}\right)-\sigma_{i}\left(U_{i}^{-}\right)\right) \frac{\left(\Delta W_{i}^{2}-\Delta t\right)}{\sqrt{\Delta t}}
\end{gathered}
$$

em que os pontos $U_{i}, U_{i}^{-}$e $U_{i}^{+}$são atualizados como:

$$
\begin{gathered}
U_{i}=X_{i}+\mu_{i}\left(X_{i}\right) \Delta t+\sigma_{i}\left(X_{i}\right) \Delta W_{i} \\
U_{i}^{-}=X_{i}+\mu_{i}\left(X_{i}\right) \Delta t-\sigma_{i}\left(X_{i}\right) \sqrt{\Delta t} \\
U_{i}^{+}=X_{i}+\mu_{i}\left(X_{i}\right) \Delta t+\sigma_{i}\left(X_{i}\right) \sqrt{\Delta t},
\end{gathered}
$$

onde $\mu_{i}\left(U_{i}\right), \mu_{i}\left(U_{i}^{-}\right)$e $\mu_{i}\left(U_{i}^{+}\right)$são os valores de $\mu$ computados nos pontos $U_{i}$, $U_{i}^{-}$e $U_{i}^{+}$, respectivamente. Já as contribuições estocásticas para esses mesmos pontos são dadas por $\sigma_{i}\left(U_{i}\right), \sigma_{i}\left(U_{i}^{-}\right)$e $\sigma_{i}\left(U_{i}^{+}\right)$, respectivamente.

\section{METODOLOGIA}

Para a obtenção dos perfis de concentração e de distribuição do tamanho dos cristais, o modelo integro-diferencial apresentado deve ser integrado. Com essa finalidade, diversas técnicas podem ser aplicadas, entre elas: Método das Diferenças Finitas, Método dos Volumes Finitos, Método dos Elementos Finitos, Colocação Ortogonal, Método dos Momentos, Método das Características, Método das Classes 
(Myerson, 1952; Rawlings et al., 1993; Rawlings et al., 2001; Costa, 2003; Shi et al, 2006, Mesbah, 2010). Para aplicar os métodos numéricos apresentados, o modelo integrodiferencial deve ser reescrito como um equivalente puramente diferencial. Neste trabalho optou-se por empregar o Método dos Momentos, que consiste na definição do momento $j$ como sendo:

$$
\mu_{j} \equiv \int_{0}^{\infty} L^{j} n(L) d L .
$$

Nessa equação, o momento zero $\left(\mu_{0}\right)$ fornece o número total de cristais no sistema; o primeiro momento $\left(\mu_{1}\right)$ fornece o comprimento total dos cristais do sistema; e a área superficial total do sistema é fornecida pela multiplicação do segundo momento $\left(\mu_{2}\right)$ pelo fator de forma em área, enquanto o volume total dos cristais é equivalente à multiplicação do terceiro momento $\left(\mu_{3}\right)$ pelo fator de forma em volume. A massa total de cristais é a multiplicação do terceiro momento com a densidade dos cristais e o fator de forma em volume (Myerson, 1952; Rawlings et al., 1993; Rawlings et al., 2001; Costa, 2003; Shi et al, 2006, Mesbah, 2010).

Com a aplicação do Método dos Momentos, a equação de balanço populacional é transformada em um sistema de equações diferenciais ordinárias. Para tanto, multiplicase o balanço de população por $L_{j}$ e se integra com relação a $L$, resultando em equações em termos dos momentos (Randolph e Larson, 1971):

$$
\int_{0}^{\infty} L^{j}\left(\frac{\partial n}{\partial t}+\frac{\partial(G n)}{\partial L}+n \frac{\partial V}{V \partial t}+D(L)-B(L)+\sum_{k} \frac{V_{k} n_{k}}{V}\right) d L=0 .
$$

Para um sistema em regime de operação batelada, considerando o crescimento independente do tamanho e que os termos de aparecimento e desaparecimento podem ser expressos em termos médios, o sistema de equações diferenciais para os três primeiros momentos é dado por:

$$
\frac{d}{d t}\left[\begin{array}{l}
\mu_{0} \\
\mu_{1} \\
\mu_{2} \\
\mu_{3}
\end{array}\right]=\left[\begin{array}{c}
B_{0}+\bar{B}-\bar{D} \\
\mu_{0} G+\bar{B}-\bar{D} \\
2 \mu_{1} G+\bar{B}-\bar{D} \\
3 \mu_{2} G+\bar{B}-\bar{D}
\end{array}\right],
$$

em que a condição inicial para cada momento é computada a partir da distribuição inicial de cristais no cristalizador, de acordo com a Equação (17). 
Nesse caso, o modelo diferencial escrito em termos dos momentos é associado ao modelo que representa o balanço de massa (concentração de cristais), de forma que um sistema de equações diferenciais determinísticos seja obtido. Assim, para trabalhar no contexto estocástico no modelo diferencial para a concentração de cristais, é inserido um termo para representar a incerteza, conforme a Equação (3). De posse do sistema estocástico, o mesmo pode ser integrado considerando as estratégias apresentadas anteriormente.

\section{RESULTADOS E DISCUSSÄO}

\subsection{Problemas Matemáticos}

Para avaliar a qualidade da solução obtida pelos métodos apresentados, considere as seguintes equações diferenciais estocásticas (Kloeden e Platen, 1999):

$$
\begin{aligned}
& d X(t)=-a^{2} \sin (X(t)) \cos ^{3}(X(t)) d t+a \cos ^{2}(X(t)) d W(t), \quad X(0)=1 \\
& d X(t)=a^{2} \cos (X(t)) \sin ^{3}(X(t)) d t-a \sin ^{2}(X(t)) d W(t), \quad X(0)=\frac{\pi}{4},
\end{aligned}
$$

cujas soluções analíticas são dadas, respectivamente, por:

$$
\begin{aligned}
& X(t)=\arctan \left(\operatorname{aW}(t)+\tan \left(X_{0}\right)\right) \\
& X(t)=\operatorname{arccot}\left(\operatorname{aW}(t)+\cot \left(X_{0}\right)\right) .
\end{aligned}
$$

A Figura 1 apresenta os perfis simulados para cada uma destas equações considerando 500 pontos de discretização, semente fixa e igual 100 na rotina rgn(seed,'v5normal') do software Matlab ${ }^{\circledR}$ (geração de número aleatórios com distribuição normal, com média igual a 1 e desvio padrão igual a zero) e o parâmetro a igual a 1.

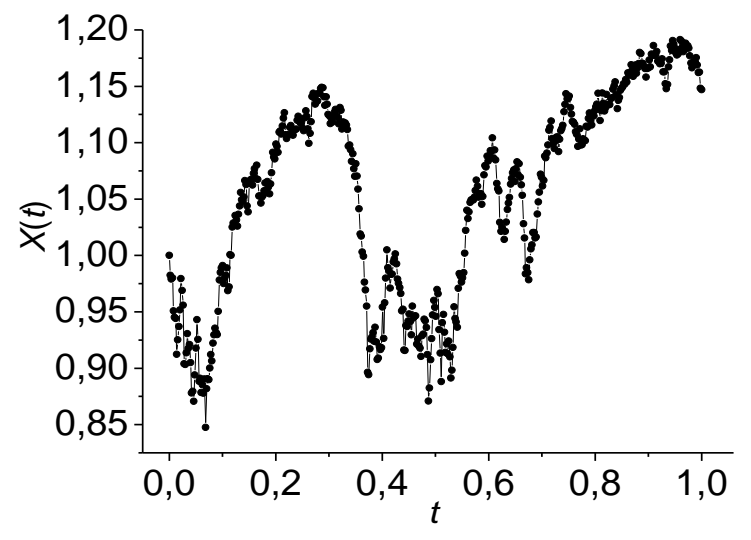

(a) EDE (Equação (20))

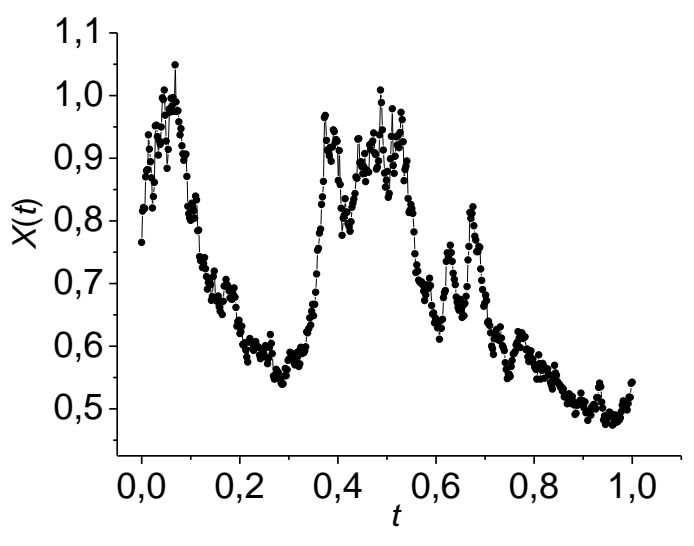

(b) EDE (Equação (21))

Figura 1: Perfil simulado considerando cada equação diferencial estocástica 
Já na Figura 2 são apresentados os erros absolutos obtidos por cada um dos métodos numéricos estocásticos em função do tempo de discretização. Para essa finalidade considera-se o número de pontos de discretização variando entre 5000 e 50000, bem como a mesma semente (100 na rotina rgn(seed,'v5normal') do software Matlab $\left.{ }^{\circledR}\right)$.

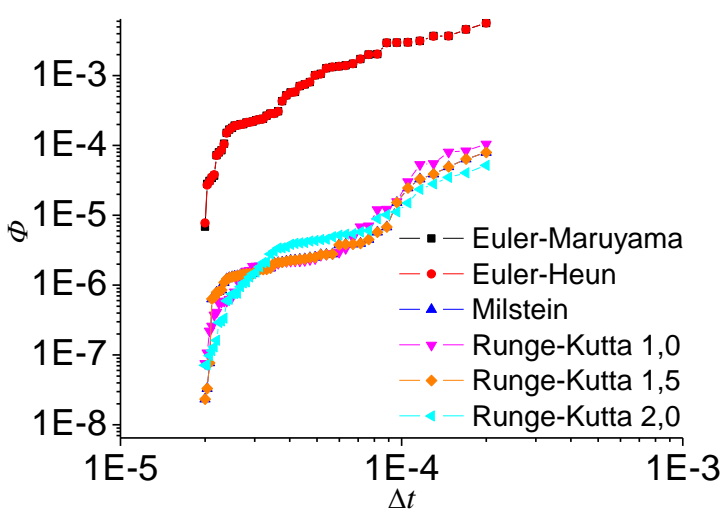

(a) EDE (Equação (20))

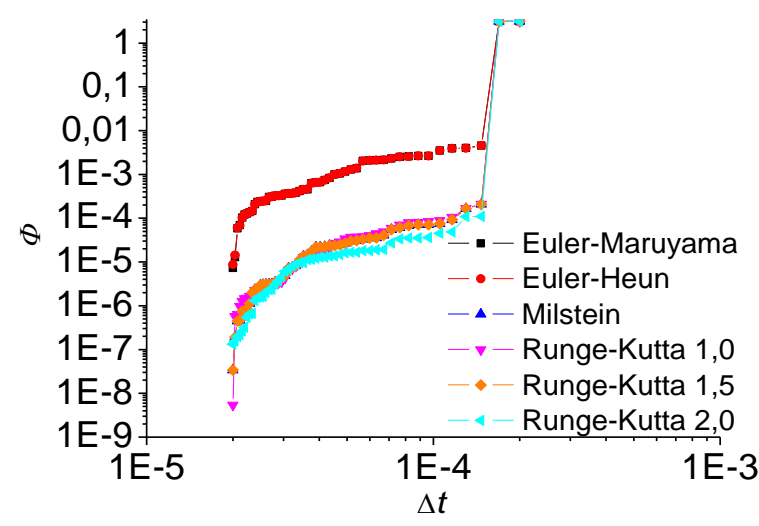

(b) EDE (Equação (21))

Figura 2: Erros absolutos em função do intervalo de integração para cada EDE

Nestas figuras observa-se, como esperado, que quanto menor for o valor do intervalo de integração, menor é o erro cometido. Além disso, para ambos os estudos de caso, observa-se perfis similares para os Métodos de Euler-Maruyama, Euler-Heun e Milstein, bem como para os Métodos de Runge-Kutta 1,0; 1;5 e 2,0.

Em termos do custo computacional (número de chamadas dos vetores $\mu$ e $\sigma$ durante o processo), tomando-se como base o Método de Euler-Maruyama, observa-se que os Métodos de Euler-Heun, Milstein e Runge-Kutta 1,0 e 1,5; requerem, a cada iteração, duas vezes mais avaliações, devido à necessidade da avaliação de um ponto extra. Já o Runge-Kutta 2,0 requer quatro vezes mais avaliações, em cada iteração, visto a necessidade da avaliação de outros três pontos durante o processo.

\subsection{Simulação do Cristalizador Batelada Não-Isotérmico}

Considere o modelo de um cristalizador batelada não isotérmico que produz cristais de sulfato de potássio, proposto por Rawlings et al. (1993) e estudado por outros autores (Rawlings et al., 2001; Shi et al, 2006\}. Matematicamente, esse modelo é definido pelo seguinte sistema de equações: 


$$
\begin{gathered}
\frac{d C}{d t}=-3 \rho_{C} k_{V} G(t) \int_{0}^{\infty} n(L, t) L^{2} d L, \quad C(0)=0,1743 \\
\frac{\partial n}{\partial t}+G \frac{\partial(n)}{\partial L}=0 \\
n(0, t)=\frac{B}{G} \\
n(L, 0)=\left\{\begin{array}{cl}
0,0032(300-L)(L-250) & 250 \mu \mathrm{m} \leq L \leq 300 \mu \mathrm{m} \\
0 & L<250 \mu \mathrm{m} \text { ou } L>300 \mu \mathrm{m}
\end{array},\right.
\end{gathered}
$$

em que $\bar{B}$ e $\bar{D}$ são nulos.

Para simular este modelo considera-se que a temperatura $T$ é representada por uma expressão linear, definida como sendo $T=(-1,95 / 3) t+50$, dada em ${ }^{\circ} \mathrm{C}$. Da mesma forma, a temperatura $T_{j}$, também dada em ${ }^{\circ} \mathrm{C}$, é definida como $T_{j}=(-3,2 / 3) t+50$. Para a situação apresentada, tem-se um sistema em que o resfriamento é controlado. Também são consideradas as seguintes equações constitutivas (Rawlings et al, 1993):

$$
\begin{gathered}
B=k_{b} \exp \left(-E_{b} / R T\right)\left(\frac{C-C_{S}}{C_{S}}\right)^{b} \int_{0}^{\infty} L^{3} n(L, t) d L \\
G=k_{g} \exp \left(-E_{g} / R T\right)\left(\frac{C-C_{s}}{C_{s}}\right)^{g},
\end{gathered}
$$

onde $E_{b}$ é a energia de ativação da nucleação; $E_{g}$ é a energia de ativação do crescimento; $b$ e $g$ são os expoentes relativos à taxa de nucleação e crescimento para a supersaturação; e $C_{s}$ é a concentração de saturação do soluto, dada por (Rawlings et al. 1993):

$$
C_{S}=6,29 \times 10^{-2}+2,46 \times 10^{-3} T-7,14 \times 10^{-6} T^{2} .
$$

A Tabela 1 apresenta os parâmetros considerados para a simulação (Rawlings et al. 1993).

A Figura 3 apresenta os perfis de concentração de cristais determinístico e estocástico considerando $\sigma$ igual a 0,005. 
Tabela 1. Parâmetros considerados para a simulação do cristalizador batelada

\begin{tabular}{cccc}
\hline$b(-)$ & 1,45 & $g(-)$ & 1,5 \\
$k_{b}\left(\min \mu \mathrm{m}^{3}\right)^{-1}$ & $1,710 \mathrm{E} 4$ & $k_{g}(\mu \mathrm{m} / \mathrm{min})$ & $8,640 \mathrm{E} 9$ \\
$E_{b} / R(\mathrm{~K})$ & 7517 & $E_{g} / R(\mathrm{~K})$ & 4859 \\
$U\left(\mathrm{~kJ} / \mathrm{m}^{2} \mathrm{minK}\right)$ & 300 & $A_{c}\left(\mathrm{~m}^{2}\right)$ & 0,25 \\
$\Delta H_{c}(\mathrm{~kJ} / \mathrm{kg})$ & 44,5 & $C_{p}(\mathrm{~kJ} / \mathrm{Kkg})$ & 3,8 \\
$M(\mathrm{~kg})$ & 27,0 & $\rho\left(\mathrm{g} / \mu \mathrm{m}^{3}\right)$ & $2,66 \mathrm{E}-12$ \\
$k_{v}(-)$ & 1,5 & $t(\mathrm{~min})$ & 30 \\
\hline
\end{tabular}

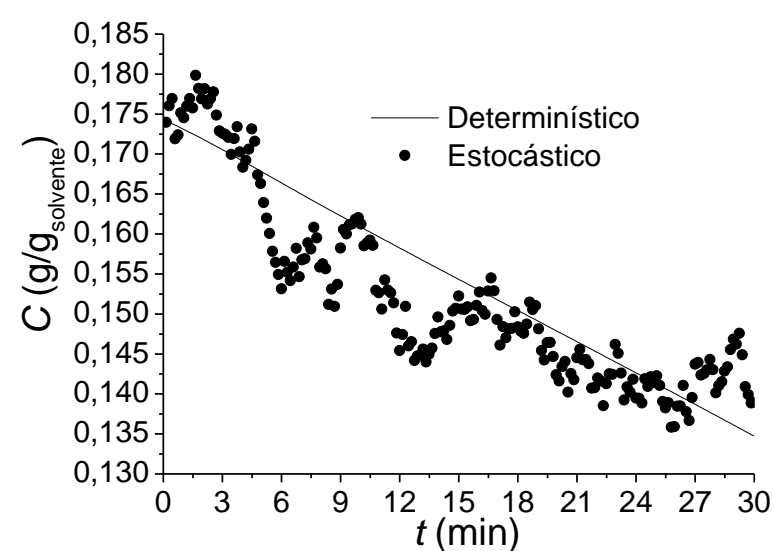

Figura 3: Perfis de concentração de cristais determinístico e estocástico considerando $\sigma$ igual a 0,005 .

Em cada um dos métodos numéricos estocásticos foram considerados 10.000 pontos de discretização, semente fixa e igual 100 na rotina rgn(seed,'v5normal') do software Matlab ${ }^{\circledR}$ (geração de número aleatórios com distribuição normal, com média igual a 1 e desvio padrão igual a zero. Os pontos experimentais sintéticos que serão apresentados na sequência foram gerados considerando a resolução do problema usando o Método de Runge-Kutta-Fehlberg, também com 10.000 pontos de discretização. Para quantificar a contribuição estocástica no perfil de concentração simulado por esse método, considerou-se $\sigma$ igual a 0,005 . Nesse caso, o vetor $\mu$ é formado pelo lado esquerdo da Equação (24), associado com o lado esquerdo do balanço de massa para os cristais (Equação (25)). Já o vetor $\sigma$ tem apenas uma posição, que é diferente de zero, a saber, a que corresponde ao balanço de massa para os cristais.

Na Figura 4, itens (a) a (f), são apresentados os perfis de concentração de cristais simulados, considerando os Métodos de Euler-Maruyama, de Euler-Heun, de Milstein, de Runge-Kutta 1,0, de Runge-Kutta 1,5 e de Runge-Kutta 2,0, respectivamente. De forma geral, em cada uma destas figuras, não se pode observar diferenças significativas entre 
os perfis simulados por cada um dos métodos considerados e os pontos experimentais estocásticos. Significa que cada um dos métodos numéricos estocásticos foi capaz de encontrar uma boa estimativa para a concentração de cristais.

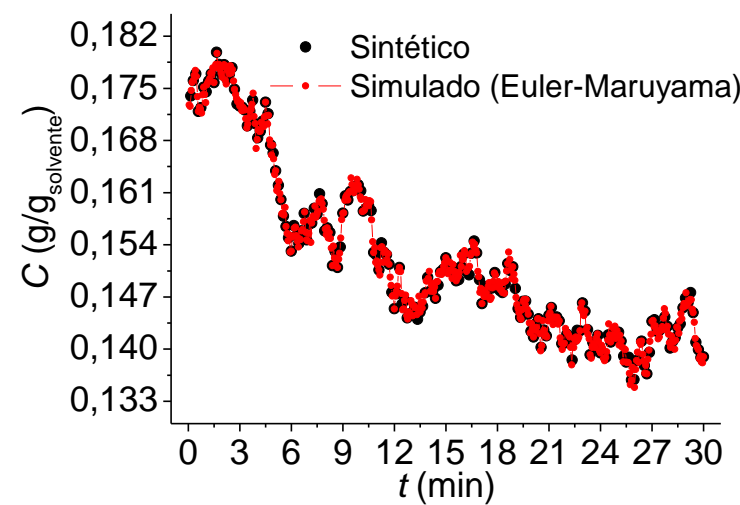

(a) Euler-Maruyama

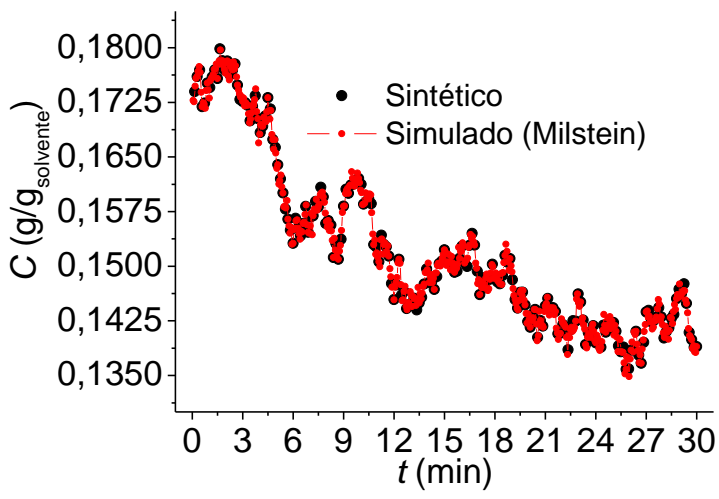

(c) Milstein

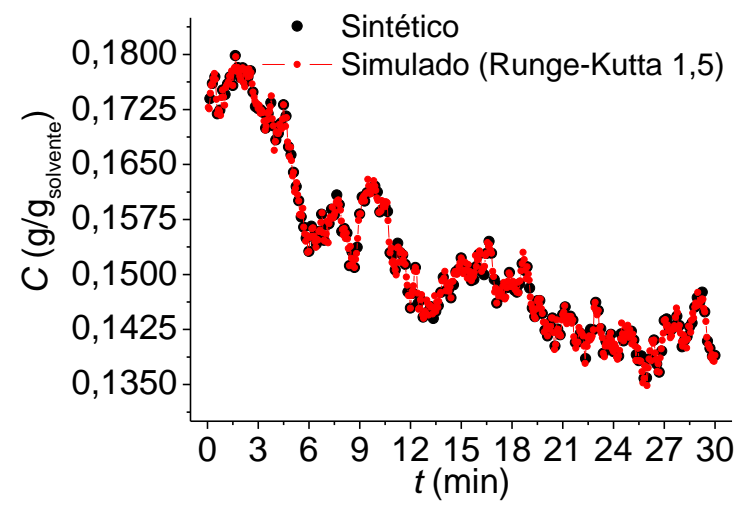

(e) Runge-Kutta 1,5

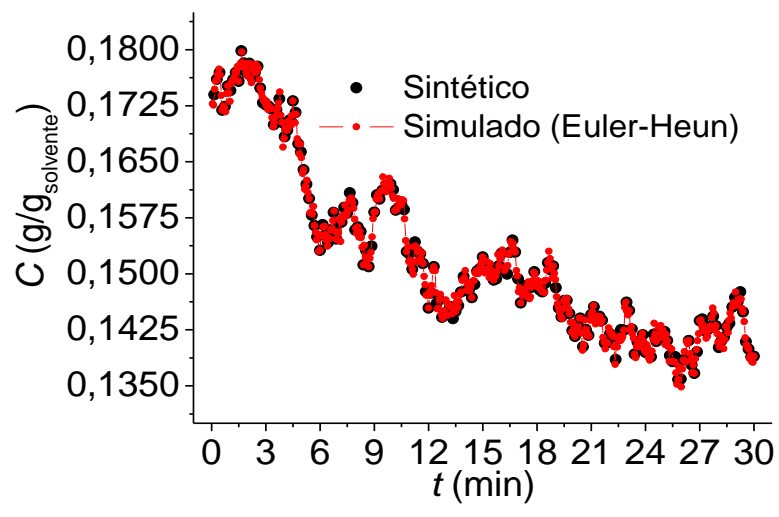

(b) Euler-Heun

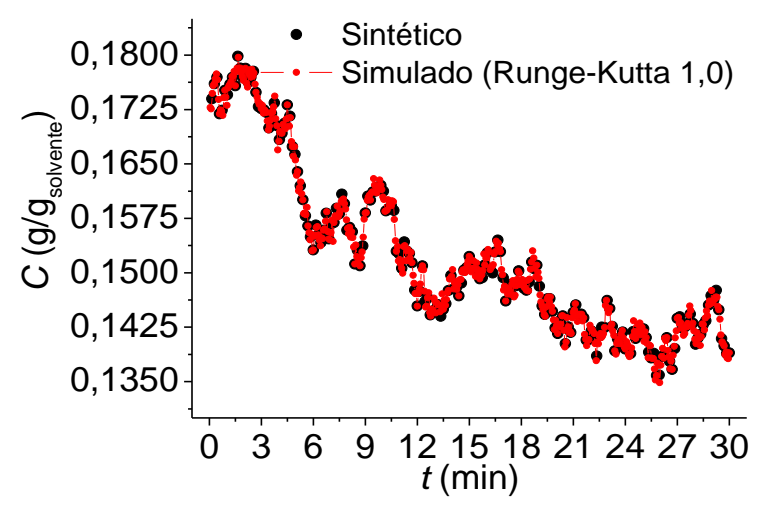

(d) Runge-Kutta 1,0

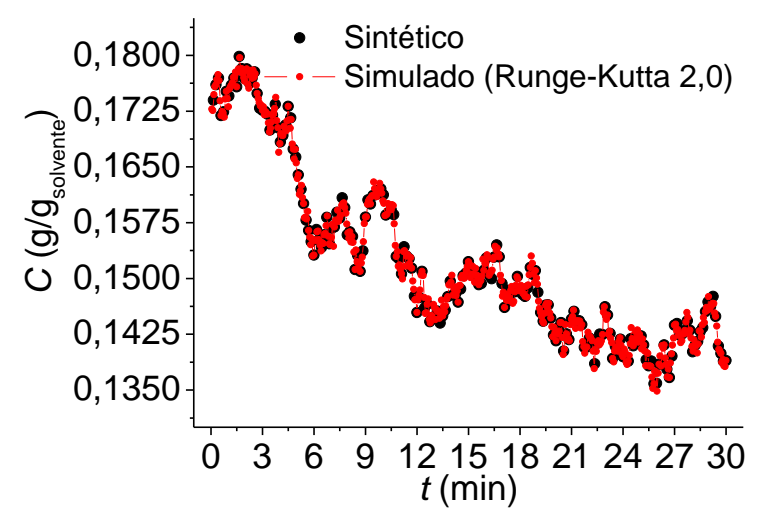

(f) Runge-Kutta 2,0

Figura 4: Perfil de concentração de cristais considerando diferentes métodos numéricos estocásticos. 
Já na Tabela 2 são apresentados o custo computacional $n_{\text {eval }}$ (número de chamadas dos vetores $\mu$ e $\sigma$ durante o processo) e o erro absoluto $\Phi$ (computado como sendo o somatório do módulo da diferença entre os resultados obtidos por cada um dos métodos estocásticos e considerando como perfil de referência o obtido considerando o Método de Runge-Kutta-Fehlberg).

Tabela 2. Custo computacional associado a cada um dos métodos estocásticos considerados para a simulação do cristalizador batelada

\begin{tabular}{ccc}
\hline Método & $\Phi$ & $n_{\text {eval }}$ \\
\hline Euler-Maruyama & $1,935 \mathrm{E}-02$ & 10000 \\
Euler-Heun & $2,510 \mathrm{E}-05$ & 20000 \\
Milstein & $1,935 \mathrm{E}-02$ & 20000 \\
Runge-Kutta 1,0 & $1,935 \mathrm{E}-02$ & 20000 \\
Runge-Kutta 1,5 & $1,935 \mathrm{E}-02$ & 20000 \\
Runge-Kutta 2,0 & $2,507 \mathrm{E}-05$ & 40000 \\
\hline
\end{tabular}

Nesta tabela pode-se observar que os Métodos de Euler-Heun e de Runge-Kutta 2,0 foram os que resultaram nos menores erros, em comparação com as outras estratégias. Em termos do custo computacional, observa-se, como esperado, que as estratégias com mais chamadas dos vetores $\mu$ e $\sigma$ são as que, naturalmente, requerem maior esforço computacional. Assim, em termos do binômio: convergência versus custo computacional, o Método de Euler-Heun foi o que apresentou melhor desempenho.

\section{CONCLUSOES}

O presente trabalho teve por objetivo simular o comportamento dinâmico da concentração de cristais em um cristalizador batelada não-isotérmico. Para isso, ao modelo determinístico foi acrescido uma perturbação para simular a estocasticidade do processo. O modelo estocástico foi resolvido considerando seis estratégias numéricas. A partir da análise do binômio convergência versus custo computacional, a abordagem que apresentou melhor desempenho foi o Método de Euler-Heun. Como proposta de trabalhos futuros, pretende-se aplicar a metodologia em outros estudos de caso, bem como estudar o problema inverso, modelado por equações diferenciais estocásticas.

\section{AGRADECIMENTOS}

Os autores agradecem ao Conselho Nacional de Desenvolvimento Científico e Tecnológico (CNPq), Coordenação de Aperfeiçoamento de Pessoal de Nível Superior 
(CAPES) e Fundação Carlos Chagas Filho de Amparo à Pesquisa do Estado de Goiás pelo suporte financeiro necessário para a realização deste trabalho. F. S. Lobato também agradece à Fundação de Amparo à Pesquisa do Estado de Minas Gerais (FAPEMIG).

\section{REFERÉNCIAS}

CARLETTI, M. Numerical Solution of Stochastic Differential Problems in the Biosciences. J. Comput. Appl. Math., 185(2), 422-440, 2006.

COSTA, C.B.B. Modelagem e Controle Ótimo do Processo de Cristalização do Ácido Adípico, Dissertação de Mestrado, Universidade Estadual de Campinas, 2003.

IVANOV, A.F.; SWISHCHUK, A.V. Optimal Control of Stochastic Differential Delay Equations with Application in Economics. Int. J. Qual. Theory Diff. Equ. Appl., 2(2), 201-213, 2008.

KLOEDEN, P. E., PLATEN, E. Numerical Solution of Stochastic Differential Equations, Springer-Verlag: New York, 1999.

LO, C.F. A Modified Stochastic Gompertz Model for Tumour Cell Growth. Computational and Mathematical Methods in Medicine, 11(1), 3-11, 2010.

MESBAH, A. Optimal Operation of Industrial Batch Crystallizers - A Nonlinear Modelbased Control Approach, Tese de Doutorado, 2010.

MYERSON, A.S. Handbook of Industrial Crystallization, $2^{\text {a }}$ ed., ButterworthHeinemann, Boston, 1952.

OKSENDAL, B. Stochastic Differential Equations: an introduction with Applications, $5^{\mathrm{a}}$ ed., Springer, New York, 1952.

RANDOLPH, A.D.; LARSON, M.A. Theory of Particulate Processes, $1^{\mathrm{a}}$ ed., Academic Press, New York, 1971.

RAWLINGS, J.B.; MILLER, S.M.; WITKOWSKI, W.R. Model Identification and Control of Solution Crystallization Process. Industrial \& Engineering Chemistry Research, 32, 1275-1296, 1993.

RAWLINGS, J.B.; SLINK, C.W.; MILLER, S.M. Control of Crystallization Processes, $2^{\text {a }}$ ed., Myerson, A.S. (ed.), Handbook of Industrial Crystallization, Butterworth-Heinemann, Boston, 2001.

RIADH, B. A Comparative Study of Numerical Simulation of Stochastic Differential Equations. Application to Stochastic Duffing-Oscillator. Int. Journal of Math. Analysis, 8(5), 229-245, 2014. 
SAITO, A.A.; IGARASHI, K.; AZUMA, M.; OOSHIMA, H. Aggregation of p-

Acetanisidide Molecules in the under-and Super-Saturated Solution and its Effect on Crystallization. Journal of Chemical Engineering of Japan, 35(11), 1133-1139, 2002.

SCHAFFTER, T. Numerical Integration of SDE: A Short Tutorial, 2009.

SHI, D.; EL-FARRA, N.H.; LI, M.; MHASKAR, P.; CHRISTOFIDES, P.D. Predictive Control of Particle Size Distribution in Particulate Processes. Chemical Engineering Science, 61, 268-281, 2006. 